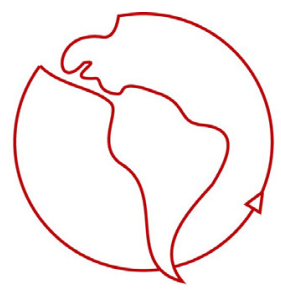

\title{
Capital Social y Estudios Sociales de la Ciencia y la Tecnología
}

Ronald Cancino

Universidad de La Frontera. Ramdom I+D+I.

rcancino@redesinnovadoras.org

\section{Resumen}

El artículo intenta una aproximación conceptual entre los Estudios Sociales de la Ciencia y la Tecnología, particularmente entre el llamado Constructivismo Social de Sistemas Tecnológicos y la Teoría del Círculo Crédito-Credibilidad, con algunos desarrollos conceptuales del Capital Social, específicamente las nociones de "Cierre de Relaciones", "Confianza Particularizada/Confianza Generalizada", "Dilema Social" y "Bien Público". Propone un modo de acercamiento de ambos campos de estudio para diseñar una estrategia teórico metodológica para el análisis y promoción de redes tecnocientíficas y tecnoeconómicas.

\section{Introducción}

El presente artículo se orienta a la generación de una metodología de análisis de redes tecnocientíficas y tecnoeconómicas intentando aunar dos series de análisis: el realizado en los llamados Estudios Sociales de la Ciencia y la Tecnología, y el realizado en torno a Capital Social. Ello en el contexto siguiente: nos interesa construir una modelización de un Sistema Regional de Innovación, específicamente para el caso de la IX Región de la Araucanía en Chile. En este contexto quisiéramos que sea entendido el ensayo. La posibilidad de realizar esta conjunción radica en lo siguiente: en ambos campos de estudio, se desarrollan conceptos que convergen en un eje común: la apertura de la "caja negra". En la explicación de la emergencia de la "verdad científica", de la "introducción de innovaciones tecnológicas" y en el "desarrollo", se trata en ambos campos de abrir y dar cuenta de las motivaciones y procesos sociales que podrían explicar esos fenómenos. Si ello es así en el ámbito de la comprensión social, también lo es en el ámbito de la intervención social: desde nuestra perspectiva, para promover la articulación entre actores económicos, científicos y sociales, se trata en primer lugar de abrirse a comprender los modos como estos entienden su dinámica y la dinámica de otros actores. Se trata en definitiva de promover interacciones reales y potenciales desde las lógicas socioculturales de los actores. Este doble ejercicio es posible desde nuestra perspectiva, aunando conceptos y estrategias de análisis provenientes de los ESCT y del Capital Social, pues es posible identificar "traslapes" conceptuales y metodológicos en ellos. En lo fundamental, nos dedicamos al análisis del llamado Constructivismo Social de Sistemas Tecnológicos y de la Teoría del Círculo Crédito-Credibilidad. Para hacer ello, seguimos el siguiente ordenamiento:

Parte I: La Apertura de la Caja Negra de la Ciencia, la Tecnología y el Desarrollo. Hacemos explícito aquí un marco desde el cuál hacer comprensible la confluencia de los ESCT y el Capital Social. Proponemos la hipótesis de la necesidad de explicar la heterogeneidad dinámica de la ciencia, la tecnología y el desarrollo.

Parte II: Traslapes Conceptuales y Metodológicos: Los Polos Normas/Estructuras; Ciencia/Tecnología. Proponemos un ordenamiento teórico en una matriz de dos ejes y cuatro polos que permiten ordenar y visualizar las confluencias conceptuales entre Capital Social y ESCT. 


\section{Parte I \\ La Apertura de la Caja Negra de la Ciencia, la Tecnología y el Desarrollo: Comprensión Social y Promoción Social.}

Tanto en el análisis social de la Ciencia y la Tecnología, como en el análisis del Capital Social, parece tenderse progresivamente a incorporar ámbitos que antes constituían campos autónomos del conocimiento: la epistemología y filosofía de la ciencia y la planificación científica y tecnológica por un lado, y la economía y la politología por otro. Quisiéramos utilizar la metáfora de "abrir la caja negra" para explicar este proceso: se trata progresivamente de comprender lo que ocurre al interior de la producción de conocimientos científicos y tecnológicos por un lado, y de comprender lo que ocurre o explica el éxito o fracaso de procesos y proyectos de desarrollo económico y social por otro. Ello permite un nuevo modo de "promoción social": se trata de promover la constitución de grupos sociales con capacidad de influir en la toma de decisiones sobre procesos o proyectos tecnológicos, de vincular la ciencia al desarrollo regional y, de fortalecer las capacidades de la sociedad civil. Estos tres fenómenos tienen un denominador común: la dotación de capital social, tanto como norma o como red de relaciones que permiten influir en la dirección de un proceso social. Veremos ello, proponiendo una unión conceptual, como el paso de la confianza particularizada (grupo social de interés en la tecnología, crédito en la ciencia) a la confianza social (clausura interpretativa y estabilización del artefacto tecnológico en la tecnología; credibilidad en la ciencia).

Existe entonces una gran profusión de discusiones y conceptos en este contexto. ¿Por qué emergen estas perspectivas?, ¿cómo podríamos comprenderlas desde nuestro contexto? Quisiéramos sostener la siguiente hipótesis: En la actualidad, podemos comprender la dinámica social como un péndulo entre la modernización y la complejización del orden social. Ello significa que existen sectores o ámbitos en la vida social que han experimentado procesos modernizadores en su seno, otros que están en proceso, otros que no han entrado en la dinámica de la modernización, e incluso algunos donde esto no es deseable. Creemos posible traducir ello en la idea de "heterogeneidad dinámica" para comprender una Región. El asunto fundamental aquí puede ser esbozado de la siguiente manera: la modernización experimentada como imperativo, más que como proceso producido endógenamente en nuestro contexto, produce la emergencia de la complejidad social, debido a la dificultad para observar los procesos socioculturales que produce tanto como efecto, pero también las dinámicas sociales que están bajo los procesos modernizadores. En este sentido, creemos que los aspectos socioculturales del desarrollo, han devenido en "ruido": fuente de incomprensión para la política pública, pero también para la teoría social. Lo sociocultural constituye así un ruido que lejos de detener las dinámicas sociales (tematizada como obstáculo o facilitador del cambio), le inyecta complejidad a esa misma dinámica.

En este contexto, se hace evidente la necesidad de "descomplejizar el ruido". Justamente este es el proceso que realizan conceptualmente los ESCT y el Capital Social. La "caja negra" no era necesaria abrirla cuando los modelos modernizadores estaban claros, pero cuando esta se pone en cuestión, por los propios efectos societales que produce, debe imperiosamente abrirse la caja negra. Así, en el análisis social de la ciencia, se pasa de los análisis mertonianos que bajo la noción de "comunidad científica" -que opera con valores y normas internas, cerradas a la sociedad- "exterioriza" los aspectos socioculturales fuera del ámbito científico, al análisis de cómo la idea de verdad e incluso falsedad científica -principio de simetría introducido por David Bloor- es un proceso de negociaciones y construcciones sociales, de manera que la ciencia pasa a ser un campo más del análisis sociológico y/o antropológico. Emerge también el análisis del círculo crédito-credibilidad de Latour-Woolgar, (donde observaremos grandes semejanzas con el Capital Social). Posteriormente, es el propio campo del Laboratorio el que es sometido a las exigencias del 
relativismo al realizarse estudios etnográficos sobre la llamada Vida en el Laboratorio (el antropólogo pionero en este ámbito es Bruno Latour).

Lo mismo ocurre con la tecnología. De ser considerada como neutral ideológica y culturalmente, pasa a ser comprendida como un producto social de un proceso social: Bijker y Pinch proponen las tesis y metodologías constructivistas sociales; paralelamente se desarrolla el análisis sistémico de Hughes, que centra su análisis en los procesos de I+D, pasando del análisis lineal de la innovación, al análisis sistémico. Finalmente, emerge el llamado actor-network, de Michael Callon, que analiza la conformación de redes de sujetos y máquinas en términos sistémicos. En este breve recorrido, hemos querido mostrar cómo progresivamente se abre la caja negra de la ciencia y la tecnología, poniéndose en el centro del análisis, tanto los aspectos relativos a redes y estructuras sociales que "configuran" y "construyen" la ciencia y la tecnología, como las normas y símbolos que en esas negociaciones se generan. Ello se traduce también en términos políticos: los ESCT han aportado conceptos y metodologías para la promoción de procesos donde los ciudadanos puedan influir en proyectos tecnológicos, así como las actividades científicas estén en sintonía con las necesidades sociales.

Similar proceso parece experimentarse con el Capital Social. Pudiendo ser comprendido como un concepto que intenta cubrir el campo vacío dejado entre el mundo académico y el mundo del desarrollo y que intenta comprender la caja negra del desarrollo (sus motivos substantivos y sus efectos substantivizados). De un lado, emerge como reacción al sociologismo, y como reacción al imperio del "rational choice". Coleman indica por ejemplo que el interés es "partir de la acción racional pero rechazando las premisas extremadamente individualistas que suelen acompañarla" (Coleman 2001:47). Se trata así de apartarse de explicaciones individualistas y "maximalistas". Similar fenómeno ocurre con Bourdieu, quien plantea que interesa analizar aquellos fenómenos que "no se dejan reducir al conjunto de las propiedades individuales que posee un agente determinado" (Bourdieu 2001: 83). En este sentido, comprendido en términos quizás metateóricos (como comprender la teoría), se trata de abordar el proceso social emergente entendido como la tensión entre individuación/desindividuación. La sociedad comienza a experimentar un retroceso de la participación en la vida cívica y una preeminencia del individuo "individualizado", lo que evidentemente convoca a redefinir los modos de comprender con nuevas categorías el fenómeno. A ello, debe agregarse otra tensión, propia del proceso modernizador: el par estructuración/desestructuración. La modernidad se construye a partir de destrucciones de lo viejo y creación de novedades de manera permanente (esa es su autolectura), de manera tal que debe comprenderse el espacio vacío dejado por esa destrucción creativa. Aún más, poco a poco comienza a aparecer como evidente que el par no funciona correctamente, pues se observan fenómenos, en el ámbito de la política pública, de dificultades para "destruir" las llamadas "estructuras y normas anquilosadas" que impiden u obstaculizan al desarrollo. Ello es indicador de la redefinición de los procesos de modernización, en lo que Lechner y el PNUD han entendido como las "paradojas de la modernización".

Pues bien, dado este escenario de dificultades o agotamiento de las perspectivas tradicionales (cambio social inducido, obstáculos y facilitadores del cambio, etc.) deben entonces generarse nuevas aproximaciones. De particular interés resulta entonces tanto comprender, pero sobretodo promover procesos de acción colectiva -fortalecer la sociedad civil, fomentar la organización, crear tejido social, creación de redes de apoyo mutuo, etc.- que no impliquen el fortalecimiento de un "actor" temido: el Estado. Putnam indica por ejemplo: "¿cómo pueden ser superados estos dilemas de acción colectiva más allá de creando algún leviathan hobbesiano?" (Putnam 2001:90). Vemos entonces que debe investigarse el capital social y promoverse tanto para comprender nuevos fenómenos creados por la propia sociedad moderna (los dilemas son justamente creaciones modernas, o dicho con mayor rigor, son imperativos modernos), como para resguardarse de la acción temida del Estado (en este sentido podríamos comprender los potentes aportes sobre la noción de jerarquía de Taylor). Todos estos momentos o procesos, creemos 
entonces pueden ser comprendidos como esfuerzos por abrir "cajas negras": la de las relaciones del individuo y grupos para la consecución de fines individuales o colectivos; la de los efectos, pero también los causales del desarrollo, y finalmente, la caja negra de la sociedad civil frente al Estado.

Quisiéramos finalizar esta sección haciendo un apunte político: la pertinencia de aunar estas perspectivas aparentemente disímiles, viene dada por lo siguiente: engendrados los ESCT en sociedades que han experimentado lo que Beck llama la "cientificación reflexiva" -emergen en ese proceso, y son justamente reflexivización relativista de la actividad científica y tecnológica- es posible hacerlo gracias a que existen dispositivos sociales que permiten la consolidación por ejemplo de Grupos Sociales Relevantes, grupos sociales -sociedad civil si se quiere- con capacidad de influencia en los diseños y aparatos tecnológicos. Ello no es tan claro en nuestros contextos, pues las más de las veces estos grupos devienen en grupos de presión sin influencia real y efectiva en la tecnología (por ejemplo, lo que ocurre con asociaciones voluntarias de defensa de grupos indígenas o de la ecología; las resoluciones en nuestro contexto vienen dadas por consideraciones políticas y económicas, escasamente por la capacidad de influencia de los grupos de presión). Por lo tanto, no es posible "importar acríticamente" estos modelos. La noción de capital social permite hacer esta "transferencia" de modelos, pues permiten pasar de reificar la capacidad de los grupos sociales para influir en la tecnología, a la necesidad de identificar los modos como construir esos grupos, es decir, a propender a la constitución de redes de influencia social en los procesos tecnológicos, que en otros contextos se conoce como "evaluación social de tecnologías".

Dicho esto entonces, nos abocamos a identificar y analizar conceptos y perspectivas de los ESCT y del Capital Social. Lo haremos mediante lo que podríamos llamar una "experimentación con conceptos", analizando la teoría Constructivista Social en Sistemas Tecnológicos y la Teoría del Círculo CréditoCredibilidad en la Ciencia.

\section{Parte II}

\section{Traslapes Conceptuales y Metodológicos: Los Polos Normas / Estructuras; Ciencia / Tecnología}

Proponemos la idea del traslape para comprender aquellos conceptos comunes existentes en el campo de análisis del Capital Social con el campo de los ESCT. Se trata de identificar esos conceptos para propender a una "dinámica de mutuo beneficio", beneficio marcado por el interés del autor de elaborar una perspectiva para analizar y promover un Sistema Regional de Innovación en el nivel de las redes entre actores científicos, económicos, políticos y sociales.

Elaboramos un diagrama que nos permite ubicar gráficamente estos traslapes. El diagrama, de "dos ejes y cuatro polos": verticalmente ubicamos el Capital Social, y horizontalmente la ciencia y la tecnología:

Capital Social: En el polo superior se ubica la comprensión del Capital Social como Normas de relaciones (el contenido de las relaciones) y en el polo inferior, la comprensión del Capital Social como Estructura de Relaciones. Así mismo, en ambos polos se encuentra "el grupo", mientras que en el punto de intersección del eje vertical con el horizontal, el individuo. En COLOR AZUL indicamos los conceptos propios del desarrollo del concepto Capital Social.

Ciencia-Tecnología: En el polo izquierdo del eje horizontal, ubicamos la tecnología, y en el polo derecho, la ciencia. Debemos decir aquí que el diagrama está construido para incorporar en él solo aquellas perspectivas que "abren la caja negra". En la intersección del eje vertical con el horizontal se encuentra el individuo: el tecnólogo y el científico solitario, mientras que hacia los polos nos acercamos a la noción de comunidad científica y grupo social de interés. En COLOR VERDE indicamos los conceptos asociados al análisis de la ciencia y en COLOR ROJO los asociados al análisis de la tecnología. 
En la página siguiente exponemos la gráfica para posteriormente analizar primero el constructivismo social de las tecnologías y luego, el círculo crédito-credibilidad en la ciencia.

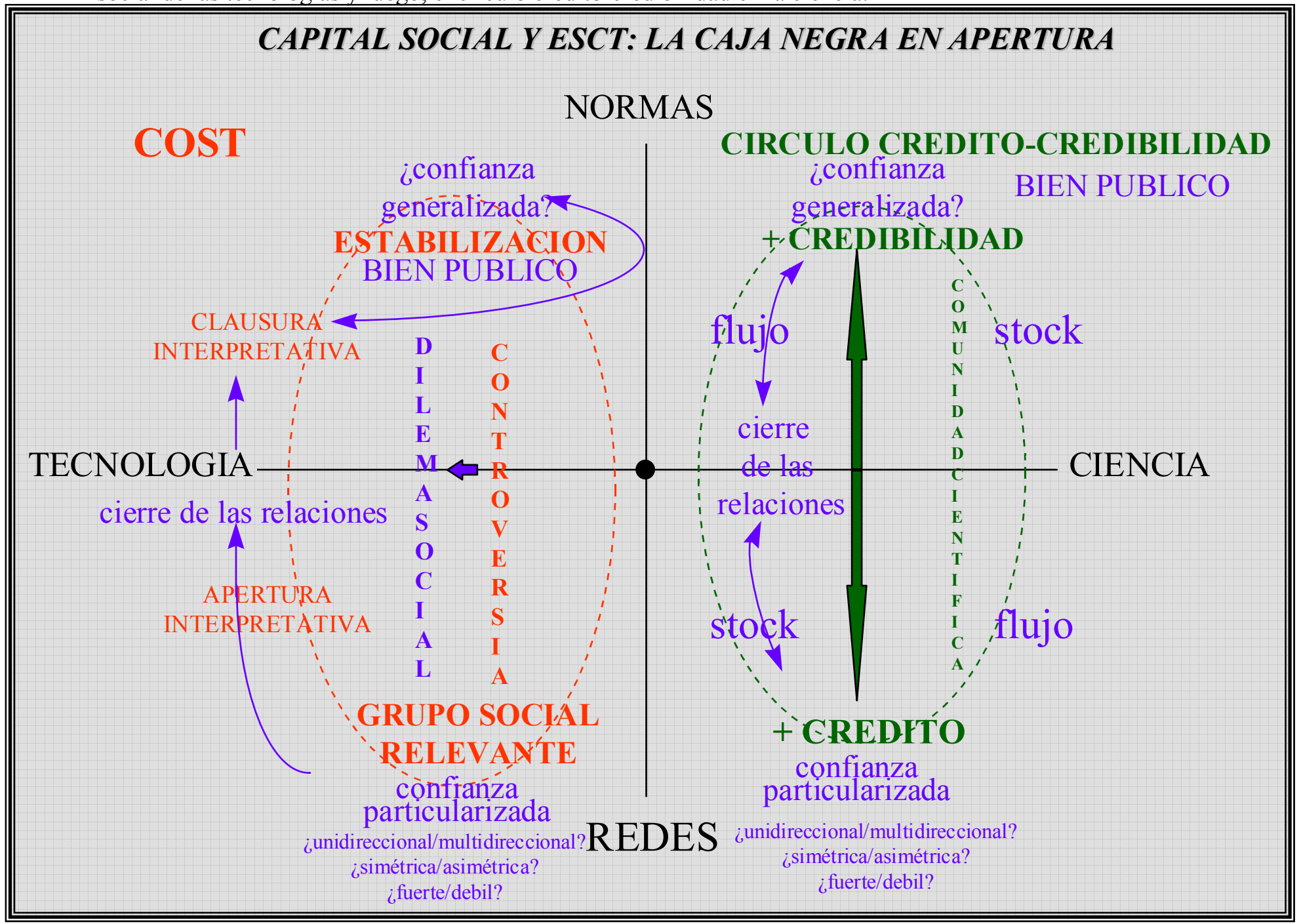

II.1. La Tecnología. La construcción social de sistemas tecnológicos: de los actores sociales relevantes como redes de confianza particularizada a la resolución de controversias tecnológicas como dilemas sociales de bienes públicos.

Trevor Pinch y Wiebe Bijker, aunando perspectivas provenientes del constructivismo social y de la sociología de la tecnología, proponen el modelo de análisis llamado COST (construcción social de sistemas tecnológicos). En lo medular, proponen que los artefactos y procesos tecnológicos son en si mismos construcciones sociales, que llegan a ser tales a través de un proceso de "variación y selección". Un artefacto tecnológico existe como controversia (un dilema social como es planteado en el análisis del Capital Social) en tanto es definida, comprendida y usada por diferentes actores sociales relevantes. Esta controversia significa que los actores comprenden, ven y usan de manera distinta el artefacto tecnológico. Hay inicialmente entonces una apertura interpretativa (el objeto tecnológico se abre a distintas 
perspectivas) y luego una clausura interpretativa (los actores ven cosas distintas). Finalmente, fruto de negociaciones entre los actores involucrados (gobierno, mercado, sociedad civil) el objeto tecnológico termina por ser redefinido: estabilización por redefinición del artefacto (efectivamente se incorporan las "demandas" al objeto, o por clausura retórica (se estabiliza mediante efectos simbólicos, solucionando otros problemas). Pues bien, en este modelo, hay una serie de interesantes "traslapes" con conceptos desarrollados en el ámbito del capital social:

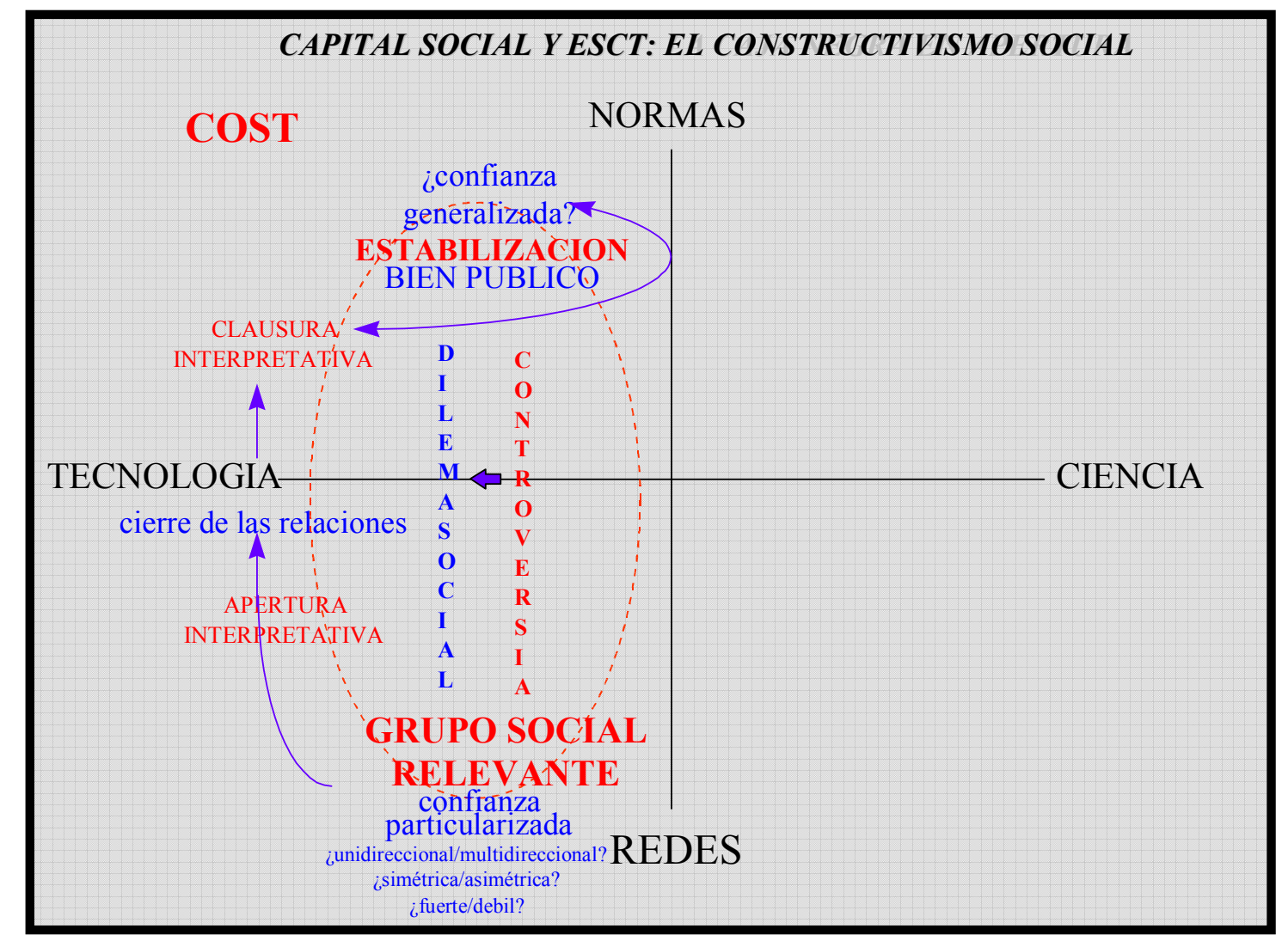

II.1.1. La Noción de Grupo Social Relevante: el GSR puede ser ubicado gráficamente como constituido en el ámbito de la estructura, o de la red. Consiste en un grupo espontáneo o preexistente, que se constituye en la confluencia de intereses comunes, que son las demandas o interpretaciones que hace del artefacto $o$ proceso tecnológico. En este sentido, podríamos decir que, en el sentido de Yamagishi y Yamagishi (en Herreros 2000:8), el GSR -como grupo- existe como "confianza particularizada", o dicho en mejores términos, se crea como una red de "confianzas particularizadas". Así mismo, hay un aspecto clave, que dice relación con el modo como se constituye el GSR: Coleman acuña el concepto de "cierre de las redes sociales" y "organización voluntaria". Creemos que se puede entender de la siguiente manera: un GSR es una "organización voluntaria", un grupo o red que existe para la consecución de un objetivo (influir en el artefacto tecnológico), pero para sostenerse y ser parte del proceso de "apertura interpretativa" debe producirse en su seno un "cierre de las relaciones sociales". Lo interesante aquí es que el cierre, que permite las normas y sanciones colectivas están orientadas hacia fuera: hacia la controversia. Siendo consecuente con la mirada constructivista, es posible participar de la controversia cuando existen mínimas 
obligaciones y normas internas al GSR. Por ello la noción de controversia se desplaza desde la estructura a las normas, de las relaciones al contenido de las relaciones.

Ahora bien, al igual que en la caracterización de las propiedades "estructurales de la red" y de las "formas de capital social", en el sentido de "que la red tenga o no cierre (closure), de que sea unidireccional o multidireccional (simplex vs multiplex), de que sea más o menos simétrica y horizontal o más o menos asimétrica y vertical, de que sus vínculos interpersonales sean fuertes o débiles, de que haya puentes locales entre redes, de la frecuencia de los contactos, de la capacidad de sanción social efectiva...." (Herreros 2000: 7), constituye un desafío teórico y empírico para comprender la construcción de un Grupo Social Relevante. Con todo, podríamos decirlo del siguiente modo: los GSR son aquellos que poseen capital social, ellos son los que pueden influir en el proceso experimentado en el orden del mercado tecnológico. Justamente, dependiendo de las dotaciones específicas de capital social, es más o menos posible influenciar el proceso de construcción de tecnologías.

II.1.2. La noción de controversia: otro de los conceptos centrales del COST, es la noción de controversia. Consiste básicamente en que una tecnología, un artefacto o proceso tecnológico se constituye, en tanto inmerso en una trama social, en un punto focal en el cual grupos con diferentes posiciones comprenden y perciben de manera distinta este artefacto. El desarrollo conceptual del capital social puede aportar aquí para comprender las dos posibles trayectorias de la controversia: puede ser estabilizada la tecnología por "clausura retórica" o por "redefinición tecnológica". La "confianza particularizada" puede ser el motor de creación y sostenimiento de GSR, pero si estos poseen un stock de capital social que les permita sostener su condición, hasta ser participes de la clausura interpretativa del fenómeno tecnológico, este artefacto o proceso tecnológico, puede ser estabilizado por "clausura retórica" (redefinición simbólica y discursiva) o por redefinición tecnológica (se incluyen en el diseño y uso del artefacto las demandas del o los GSR. Las nociones de "bien público" y "dilema social" pueden aportar sustantivamente aquí. Dado que el proceso de la controversia (apertura/clausura interpretativa) necesariamente se traduce en estabilización de la tecnología, la pregunta clave radica en la capacidad que tienen los grupos sociales relevantes de traducir esa controversia en un "dilema social" y en un problema de "bien público". Creemos que depende del capital social que puedan movilizar los GSR en el proceso de la controversia, pues si un o unos GSR poseen un stock de capital social, o si entendido como flujo logran acrecentarlo, estarán en mejores condiciones de traducir la controversia en "dilema social" y hacer ver que la tecnología en cuestión constituye un "bien público". Veamos ello:

II.1.2.1. Controversia tecnológica y clausura retórica: una clausura retórica existe cuando una controversia es resuelta discursiva o simbólicamente, es decir, cuando el artefacto tecnológico en si mismo mantiene sus características iniciales. Esto, desde la perspectiva del capital social, puede ser entendido de la siguiente manera: el "bien público" (la tecnología) no ha logrado ser posicionado como "dilema social", o el "bien público" ha sido apropiado por el GSR que poseía más capital social. En ambas situaciones, la tecnología se mantiene como tal y no ha variado. Un aspecto interesante de analizar aquí puede ser el flujo del capital social y la transformación posible desde la confianza particularizada a la confianza generalizada. Teóricamente podríamos decir que en contextos de clausura retórica, la confianza particularizada que permite como decíamos el inicio del proceso, no logra devenir en confianza generalizada, pudiendo incluso producirse un retroceso en los stocks de capital social. Ello evidentemente es materia de investigación empírica.

II.1.2.2. Controversia y estabilización por redefinición de la tecnología como "bien público": cuando se produce una estabilización por redefinición de la tecnología, significa decíamos que se influye efectivamente en el artefacto o proceso tecnológico de acuerdo a la convergencia (clausura interpretativa) 
de los distintos GSR. Esta situación, creemos puede ocurrir cuando los GSR han logrado no solo mantener el stock de capital social como red o estructura, sino también como normas (sus valores se generalizan en la sociedad): han logrado en esa ampliación del CS de transformar la controversia en un "dilema social": se hace evidente socialmente que hay contradicciones entre los intereses públicos y los privados, y una vez reconocido ello, uno, o varios GSR logran influir en la tecnología. En ello vemos que se generaliza en la sociedad la idea de "bien público". Al igual que en el caso anterior, quizás lo más interesante es, si en este proceso "exitoso", la confianza particularizada que permite iniciar el proceso de la controversia se traduce en "confianza generalizada" para él o los GSR que terminan por influir en la estabilización. Ello es materia de investigación empírica.

\section{II.2. La Ciencia. El círculo crédito-credibilidad: de la confianza particularizada al interior de una comunidad científica a la credibilidad como confianza social del científico.}

Nos interesa aquí analizar una perspectiva teórica derivada de los análisis de Pierre Bourdieu sobre el campo científico. Bruno Latour (pionero en los análisis etnográficos de Laboratorio) y Steve Woolgar (sociólogo proveniente de la escuela etnometodológica) proponen un modelo de análisis que quiere superar algunos inconvenientes del análisis del campo científico de Pierre Bourdieu, pero da continuidad a algunos de sus principios. De un lado, adopta la idea de que la comunidad científica no se mueve sencillamente por los principios de racionalidad y desinterés, pero crítica algunas tautologías de Bourdieu, fundamentalmente sobre la noción de "autoridad científica" (posee capital simbólico porque lucha con su capital simbólico). Frente a esta noción, proponen el concepto de credibilidad: los científicos "invierten" en credibilidad para recibir créditos y aumentar la credibilidad. El crédito, son los mecanismos para continuar y profundizar la actividad científica, que lleva al investigador a aumentar su credibilidad, y así sucesivamente.

Dicen Latour y Woolgar: "Supongamos que los investigadores sean inversores de credibilidad. Ello trae como resultado la creación de un mercado. La información ha adquirido un valor, y autoriza a otros investigadores a producir la información que permita el retorno del capital invertido... los investigadores invierten allí en donde ésta tiene más chances de ofrecer un retorno más alto. Su evaluación de esas fluctuaciones explica, al mismo tiempo, la referencia que hacen los investigadores a los "problemas interesantes", temas que "dan beneficio", "buenos métodos" y "colegas en quienes se puede tener confianza"...."(1995).

Se trata entonces de lo siguiente: la lógica de la actividad científica estaría regulada no por los principios mertonianos del desinterés y el universalismo, sino por la centralidad de la noción de crédito. El crédito, no consiste solo en la recompensa -económica por ejemplo-, sino en tanto mecanismo que permite aumentar la credibilidad: "se tiene la impresión de que, aunque la noción de crédito como recompensa es importante, es un fenómeno secundario. Por ejemplo, solamente al final de una extensa carta pidiendo sustancias, proponiendo experimentos y sugiriendo ideas, Herbert daba las gracias por la acogida en una reunión reciente y añadía: "Por lo que se refiere a tu trabajo anterior... ciertamente mereces todo el crédito por estas observaciones conductuales astutas y tempranas". El crédito, la inversión que hace el científico tiene una orientación: aumentar circularmente la credibilidad frente a sus pares, para con ello obtener más crédito. Se trata de acelerar el ciclo.

Pues bien, sobre estas nociones básicas, es posible hacer varias observaciones desde el análisis del capital social, particularmente de las nociones que trabajamos para el caso de la COST, (pues como dijimos al inicio del texto, nos interesa elaborar una perspectiva metodológica para comprender y promover redes tecnocientíficas y tecnoeconómicas): cierre de relaciones, bien público, confianza particularizada y 
confianza generalizada. Lo haremos en tres ejes: stock/flujo; individual/colectivo y el problema del bien público.

II.2.1. El Capital Social como stock y como flujo en el círculo crédito credibilidad: si seguimos la lógica de Latour Woolgar, el capital social debe ser entendido aquí como la capacidad que busca el investigador por aumentar su stock de capital social, de manera que este le permita aumentar la velocidad del círculo: el investigador tiene en un momento dado un stock de capital en tanto inserto en una red de investigadores que comprende como "su comunidad científica" (un departamento, una facultad, un colegio o asociación). Se trata para él de aumentar su stock, es decir, de transformarlo en flujo: al convertir el stock en flujo, se puede comprender el círculo.

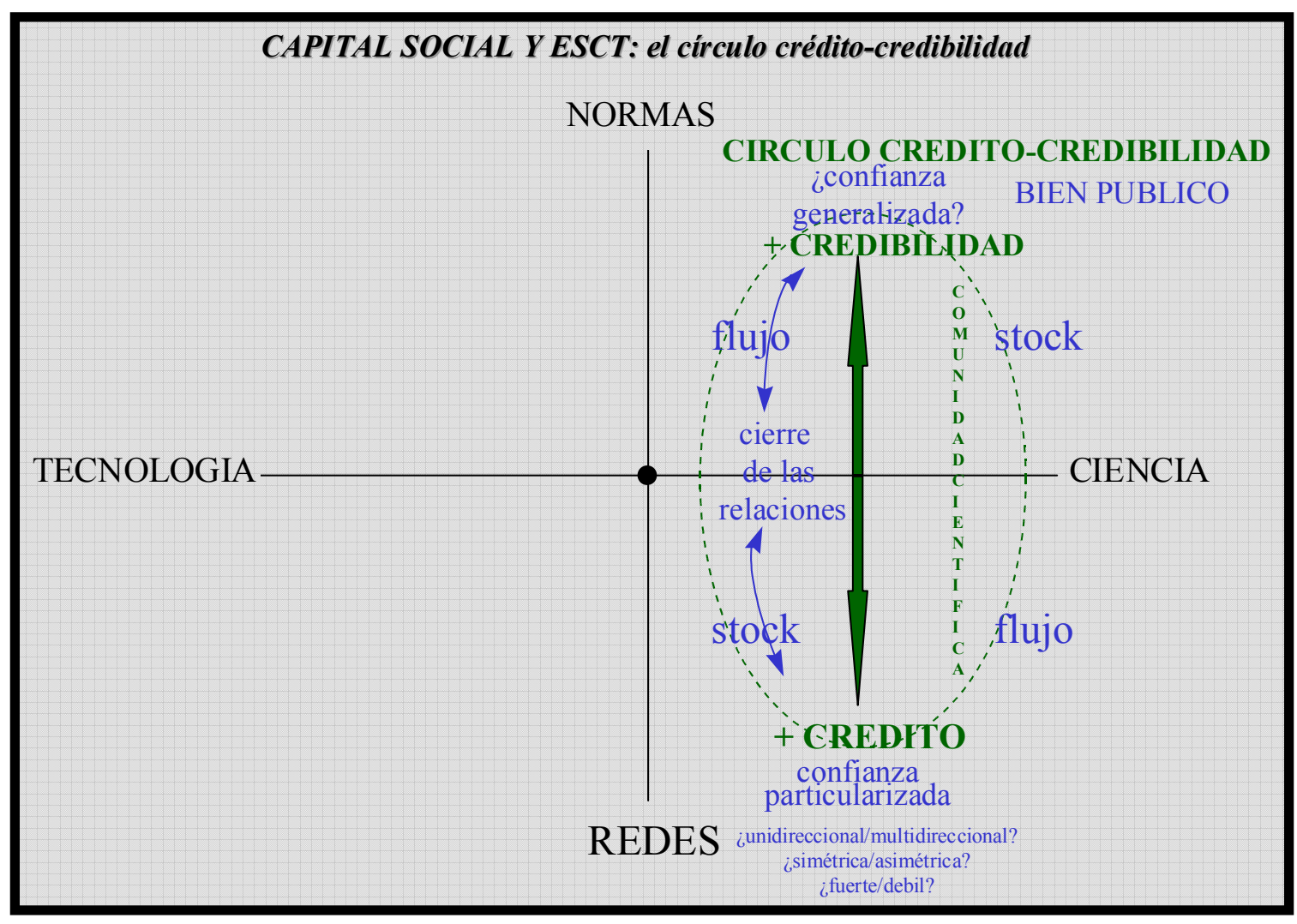

Lo interesante aquí es que el investigador inserto en una red (capital social como red o estructura) busca aumentar su credibilidad, que podemos entender como el aumento de la creencia en él como sujeto, o grupo de sujetos que comparte las normas y valores de su comunidad (todos buscan el aumento de la credibilidad). Así, la dinámica stock-flujo, puede ser entendida como el círculo red-norma.

II.2.2. Capital Social individual y colectivo: el investigador es inicialmente un investigador solitario, que utiliza sus redes científicas para aumentar su credibilidad para obtener más crédito. Aquí, el capital social es inicialmente un recurso individual, pero para insertarse en la red -comunidad- debe ser participe de ella, de manera que para sostener el capital social individual, requiere del capital social colectivo. Podríamos decirlo del siguiente modo: la comunidad no es simplemente un agregado de investigadores individuales, sino que se cierra mediante el "objeto" de estudio: el contenido de la practica científica (disciplina, subdisciplina, sector de aplicación) es el que media entre el capital social individual y el capital social 
colectivo. Así, el cierre de las relaciones opera como medio para aumentar la velocidad del círculo crédito-credibilidad.

II.2.3. Capital Social y crédito-credibilidad como "bien público": el investigador busca el reconocimiento para aumentar su credibilidad. Si consideramos que los productos de su actividad son "bienes públicos" por ejemplo, en nuestro contexto, financiados las más de las veces por el representante máximo del bien público, el Estado-, entonces el "cierre de las relaciones" que hace el investigador, es posible mediante la existencia de una "confianza particularizada" ("colegas en quienes se puede tener confianza"). Ahora bien, al aumentar la credibilidad, esta confianza se transforma progresivamente en "confianza generalizada": se trata de un reconocimiento social incluso externo a la propia comunidad. Este proceso resulta estratégicamente muy potente para promover procesos de articulación o generación de redes entre comunidades científicas y comunidades "no científicas": en tanto esas relaciones resulten beneficios para el círculo crédito-credibilidad, podríamos esperar un alto interés en participar en ellas.

\section{Conclusiones: Hacia el Análisis de Redes Tecnocientíficas y Tecnoeconómicas}

Quisiéramos esbozar aquí algunas conclusiones de la revisión y "experimentación conceptual" realizada. Las hacemos pensando en un diseño metodológico con un objetivo doble: analizar y promover redes en el contexto del estudio de un Sistema Regional de Innovación.

En primer lugar, nos interesa destacar que es posible producir el acercamiento o conjunción de las perspectivas analizadas, por un motivo fundamental: en nuestro contexto, se trata de procesar críticamente perspectivas teóricas. Decimos críticamente porque la emergencia del campo del Capital Social, como de los ESCT se relaciona con los efectos que los propios procesos modernizadores producen en la sociedad. Por ello, en un contexto como el nuestro, que indicamos brevemente como un tránsito entre la modernización y la complejización del orden social, donde los aspectos socioculturales del desarrollo han devenido en ruido, no es posible reificar los modelos: no podemos por ejemplo dar por sentada la existencia dinámica de Grupos Sociales Relevantes, o la existencia clara y por todos reconocida de "Dilemas Sociales". Se trata de comprender su existencia como una heterogeneidad dinámica. Por ello, el análisis realizado parece permitir tanto la "dinámica de mutuo beneficio" como la comprensión y promoción de redes articuladas de actores científicos, económicos y políticos.

En segundo lugar, respecto de la comprensión o el análisis, nos parece que es posible pensar lo sistemas tecnológicos no como existentes per se, sino como inmersos en una trama de relaciones socioculturales, donde la noción de controversia puede permitir identificar cuáles son las principales controversias que enfrenta, o debería enfrentar un Sistema Regional de Innovación, y el modo cómo operan en su interior los Grupos Sociales Relevantes. Así mismo, estos GSR (empresarios, ONGs, agrupaciones voluntarias, académicos, organismos públicos) operan con lógicas socioculturales que es necesario comprender, pues como muestra el COST estos existen en tanto ven y comprenden diferencialmente los procesos tecnológicos (en el ámbito forestal o agrícola, por ejemplo). Se trataría entonces de comprender cómo se producen las confianzas particularizadas en esos grupos que permiten sostenerlos como tales, y analizar el modo posible (al estilo de la prospectiva o la diagnosis) de potenciar esas confianzas para traducirlas en redes. Similar cosa ocurre en el ámbito científico: se trata de identificar las principales "controversias científicas" de un sistema regional, de cómo estas están o no están articuladas a "controversias tecnológicas", y de cómo opera la lógica del crédito-credibilidad en el sistema académico regional, cómo se producen los "cierres de relaciones" (por ejemplo, en lo estrictamente académico, que podría indicar una clausura hacia las necesidades o demandas regionales, o en una apertura al mundo regional). 
Con lo anterior, creemos es posible analizar los modos posibles de propender a insertar una dinámica de articulaciones de las lógicas tecnocientíficas y tecnoeconómicas a necesidades sociales y económicas regionales y locales. En este sentido, pensamos en una metodología proactiva: comprender para promover, o comprender promoviendo. Para ello, creemos resulta clave la noción de "bien público": la tecnología y la ciencia son bienes públicos, en tanto son producidas y operan insertos en una comunidad regional, pero al no ser reconocidas como tales, se trataría de identificar y promover "dilemas sociales": poner en la discusión regional aquellos temas que la propia comunidad tecnocientífica y tecnoeconómica considera relevantes.

Dicho en síntesis, se trata de identificar los mecanismos a través de los cuales operan las confianzas particularizadas en redes tecnocientíficas (científicos, técnicos, administradores universitarios) y en redes tecnoeconómicas (empresarios chicos y grandes, ingenieros y técnicos) y como políticamente puede pasarse a la promoción de una confianza social para la producción de innovaciones tecnológicas y conocimientos científicos.

\section{Bibliografía}

Bourdieu, P. 2001. El capital social. Apuntes provisionales. Zona Abierta 94-95: 83-87.

Coleman, J. 2001. Capital social y creación de capital humano. Zona Abierta 94-95: 47-81.

Herreros, F. y Francisco, A. 2001. Introducción: el capital social como programa de investigación. Zona Abierta 94-95: 1-46.

Latour, B. y Woolgar, S. 1995. La vida en el laboratorio: La construcción de los hechos científicos. Madrid: Alianza Universidad.

Putnam, R. 2001. La comunidad próspera. El capital social y la vida pública. Zona Abierta 94-95: 89-104.

\section{Cómo citar este artículo}

Cancino, R. 2006. Capital Social y Estudios Sociales de la Ciencia y la Tecnología. Revista Mad 15: 104114. 